\title{
Posterior association cortex and visual pattern discrimination in the rat
}

\author{
WILLIAM F. McDANIEL, LINDA D. WILDMAN, and RAYMOND H. SPEARS \\ Georgia College, Milledgeville, Georgia 31061
}

\begin{abstract}
Rats with parietal, temporal, or frontopolar cortex lesions and nonoperated controls were trained on a complex visual pattern discrimination. The rats with temporal association cortex damage committed significantly more errors than those with parietal association cortex damage and nonoperated controls. These results complement earlier findings of a dissociation between the effects of temporal and parietal association cortex lesions.
\end{abstract}

Recent anatomical (McDaniel, McDaniel, \& Thomas, 1978) and behavioral investigations have suggested that there are two dissociable regions comprising the posterior association cortex of the rat. Using Lashley's cortical maps (Lashley, 1941, p. 85) as a guide for lesion placement, it has been shown that parietal association cortex damage is associated with deficits on the Lashley III maze, the HebbWilliams maze, a spatial reversal task, and a complex visual pattern discrimination. Parietal lesions do not, however, disrupt the learning of a black-white discrimination reversal task, a simple pattern discrimination, and the relearning of a spatial reversal following preoperative training (Boyd \& Thomas, 1977; McDaniel \& Thomas, 1978; Thomas \& Weir, 1975; Weir \& Thomas, 1975). Lesions of the temporal association cortex produce deficits which are similar to those seen in the tree shrew (Tupaia glis) and the squirrel (Sciurus carolinesis) following destruction of the temporal association cortex (Diamond \& Hall, 1969; Killackey, Diamond, Hall, \& Hudgins, 1968; Killackey, Snyder, \& Diamond, 1971; Killackey, Wilson, \& Diamond, 1972; Snyder, Hall, \& Diamond, 1966). These deficits include impaired performance on a black-white discrimination reversal task and the learning of both simple and complex visual pattern discriminations. This deficit is not one of a generalized nature, since rats with temporal cortex damage show no deficits in maze learning and on the learning of spatial reversals (Boyd \& Thomas, 1977; McDaniel \& Thomas, 1978).

Boyd and Thomas (1977) found that there is an apparent similarity between the effects of parietal and temporal cortex destruction on the acquisition of

\footnotetext{
This study was supported in part by a Georgia College Faculty Research Grant, Grant 8289 from the Penrose Fund of the American Philosophical Society, and funds from the Georgia College Foundation and Alumni Association to the first author. It is a pleasure to thank Dr. Roger K. Thomas and Ms. Kar Burns of the Department of Psychology, University of Georgia, for assistance with the histology. Reprint requests should be sent to the first author.
}

a complex visual pattern discrimination (i.e., upright vs. inverted triangles enclosed by annuli) following the acquisition of a geometrically less complex pattern discrimination (i.e., upright vs. inverted triangles). However, the nature of this deficit remains unclear. Parallel to an explanation of a similar deficit seen in tree shrews with striate damage on this task (Killackey \& Diamond, 1971), it might be suggested that the parietal lesion deficit represents an impairment of visual attention that results in a greater distraction by irrelevant stimuli. This view receives further credence from several neurophysiological studies that have demonstrated that the activity of neurons in the parietal association cortex is associated with several visuo-attentional mechanisms in rhesus macaques (Mountcastle, 1976) and cats (Robertson, 1976; Robertson, Mayers, Teyler, Bettinger, Birch, Davis, Phillips, \& Thompson, 1975). Because of the close anatomical relationship between the temporal region and striate cortex (Montero, Bravo, \& Fernández, 1973) and the lateroposterior thalamic nucleus (Hughes, 1977; McDaniel et al., 1978), it might be argued that the temporal deficit represents an impairment of visual information processing mechanisms. The present experiment was designed to compare rats with either parietal or temporal assiociation cortex lesions on the acquisition of a complex visual pattern discrimination.

\section{METHODS}

\section{Subjects}

Twenty-four male and female hooded rats (Long-Evans strain purchased from Simonsen Laboratories, Gilroy, California) ranging in age from 90 to 110 days at the time of surgery were used. These rats were pseudorandomly assigned to the four conditions to receive parietal, temporal, or frontopolar ablations, or to serve as nonoperated controls. The restrictions imposed on randomicity were that each group have equal numbers of males and females and that each group have equal ns. One animal that had received a temporal cortex ablation died prior to completion of the task. The animals were maintained on a reverse dark-light cycle with light onset at 7:00 p.m. and offset at 7:00 a.m. All testing was done during the dark phase. 


\section{Surgery and Ablation Analysis}

All surgery was performed with the rat anesthetized with Equithesin $(.30 \mathrm{ml} / 100 \mathrm{~g}$ body weight). Clean surgical techniques were used. After a midline incision was made and the dorsal cranium exposed, the skull was drilled with a trephine of the size and at the coordinates used by McDaniel and Thomas (1978). After the underlying cortical matter was aspirated, the scalp was sutured.

At the end of the experiment, the animals were deeply anesthetized and perfused intracardially with $50 \mathrm{cc}$ of $.85 \%$ saline followed by $50 \mathrm{cc}$ of $10 \%$ Formalin. The brains were removed, stored in Formalin, and later photographed from the dorsal view. The photographs were superimposed on Lashley brain diagrams and the boundaries of the lesion traced. The Lashley brain diagrams were then used to determine the cortical areas destroyed and the percentage of total cortex ablated. Ablation percentages were computed by the dot-grid method (Thomas \& Peacock, 1965). The brains of the animals that had received either temporal or parietal cortex ablations were embedded in celloidin, sectioned at $30 \mu \mathrm{m}$, mounted on slides and stained with cresyl violet.

\section{Apparatus and Procedure}

The apparatus was the same as the two-choice alley previously described by McDaniel and Thomas (1978) and consisted of a startbox, a choice arena, and two parallel goalboxes. The startand goalboxes were constructed to be interchangeable, with the rear wall constructed of Plexiglas. The visual stimuli used consisted of black upright and inverted equilateral triangles (8-cm sides) centered on a background of either horizontal or vertical alternating black and white stripes $(1 \mathrm{~cm})$. The stimuli were placed at the rear of the goalboxes, and each was illuminated with a $25-\mathrm{W}$ light bulb.

Ten days following surgery, the animals were food deprived for $48 \mathrm{~h}$, and on Day 12 they were adapted to the apparatus by being placed in each of the goalboxes with both food dishes baited. On Days 13 and 14, they were placed in the startbox and allowed to explore the apparatus for $10 \mathrm{~min}$ each day with six 45-mg Noyes food pellets located at the rear of each goalbox. Alley running was conditioned starting on Day 15 by giving the animals 40 trials in which both goalboxes were baited with two Noyes food pellets. Following the 40 pretraining trials, training began with the relevant cue for discrimination being orientation of the triangles. The stripe orientations were irrelevant cues. Hence, the animal faced four discriminations: (1) upright triangle, vertical stripes vs. inverted triangle, vertical stripes, (2) upright triangle, vertical stripes vs. inverted triangle, horizontal stripes, (3) upright triangle, horizontal stripes vs. inverted triangle, vertical stripes, and (4) upright triangle, horizontal stripes vs. inverted triangle, horizontal stripes. Each of these four combinations was presented five times every two sessions, and each session consisted of 10 trials. One-half of the animals from each group were trained to approach the upright triangle and one-half were trained to approach the inverted triangle. The positions of the correct stimuli were randomized according to a Gellerman series (1933). Criterion for learning consisted of the animal scoring at least $80 \%$ correct responses on 2 consecutive days, and the animals were trained using a correction procedure previously described (Boyd \& Thomas, 1977; McDaniel \& Thomas, 1978; Snyder \& Diamond, 1968). A ceiling of twice the number of trials required by the first two-thirds of the animals to reach criterion was used. Therefore, training was terminated for animals that had not learned the discrimination after 840 trials. Errors were signaled by onset of a $45-\mathrm{W}$ light and a buzzer for $1 \mathrm{sec}$, and the rats were trained via an experimenter-blind procedure.

\section{RESULTS}

An analysis of variance was computed on the errors to criterion (or ceiling) after a Hartley F max analysis (Gehring, 1978) revealed that, although three rats with temporal lesions and two with frontopolar lesions failed to learn the discrimination within 840 trials, assumptions of homogeneity of the group variances had not been violated ( $\mathrm{F}$ max, calc, .05 = 2.94; F max, crit, $.05=13.7$ ). Results of the analysis of variance on the error score data summarized in Table 1 showed that the between-groups effect was significant $[F(3,19)=4.03, p<.025]$. NewmanKeuls analysis indicated that the temporal group committed more errors than the group with parietal ablations and the nonoperated controls (ps $<.05$ ). No other group differences were significant, and hence the frontopolar group was intermediate between the temporal group and the parietal and nonoperated groups.

A possible measure of distraction by irrelevant stimuli inherent in this study might be the number of errors scored on the orientation of triangle discrimination when the backgrounds were alike (paired horizontal stripes or paired vertical stripes) compared with the number of errors made when the background stimuli were different (horizontal stripes for one background paired with vertical stripes for the other background). It was suggested that different irrelevant background cues might be more distractive than background cues that were the same and that animals with attentional impairments would evince deficits on the latter discrimination. Therefore, an additional three-way analysis of variance (Lesion Group by Stimulus Background by Blocks of Trials) with repeated measures on two factors and with appropriate adjustments for unequal ns was conducted on the number of errors made on discriminations when the backgrounds were identical (i.e., both horizontal or both vertical stripes) and when they were different (i.e., paired horizontal and vertical stripes) on the first 100 trials (Block 1) and the final 100 trials (Block 2). Errors from the first and the final 100 trials were used in this analysis because there was a variable number of total trials trained

Table 1

Mean Trials to Criterion and Errors Committed for the Four Groups

\begin{tabular}{|c|c|c|c|c|c|c|c|c|}
\hline & \multicolumn{2}{|c|}{ Nonoperated } & \multicolumn{2}{|c|}{ Frontopolar } & \multicolumn{2}{|c|}{ Parietal } & \multicolumn{2}{|c|}{ Temporal } \\
\hline & Mean & SD & Mean & SD & Mean & SD & Mean & SD \\
\hline Trials to Criterion & 423 & 127.54 & 577 & 216.39 & 420 & 123.77 & 718 & 175.27 \\
\hline Errors & 207 & 68.48 & 278 & 109.36 & 202 & 63.81 & 352 & 77.96 \\
\hline
\end{tabular}



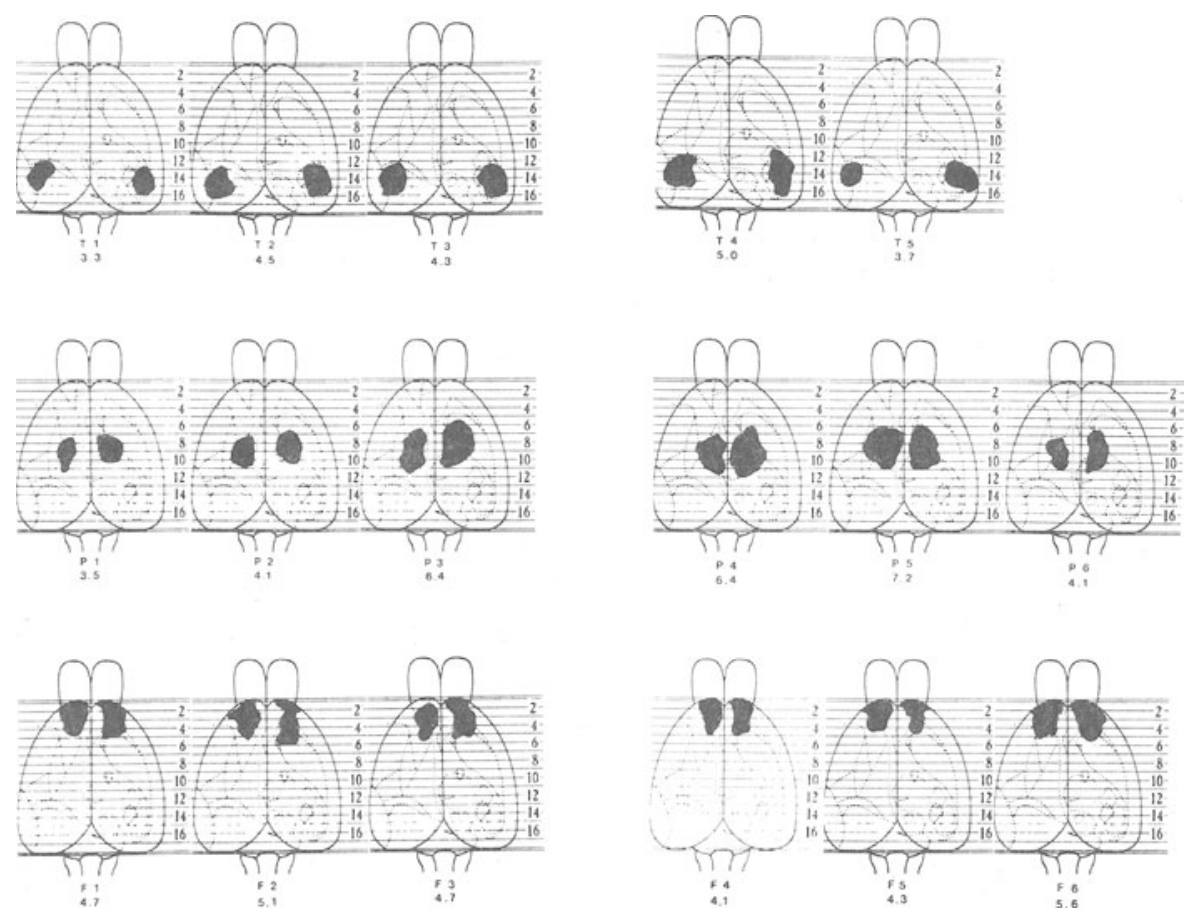

Figure 1. Reconstructions of the cortical ablations from the dorsal view. Black areas indicate lesion sites and the lower numbers below each brain indicate the percentage of total cortex ablated. Abbreviations: $T$, temporal cortex; $P$, parietal cortex; $F$, frontopolar cortex.

among the animals. Results of this analysis showed, as predicted, that more errors were made on the orientation of triangle discrimination when the background stimuli were different $(\bar{X}=23.17)$ than when the background stimuli were the same $(\overline{\mathrm{X}}=$ 21.75) $[\mathrm{F}(1,20)=12.01, \mathrm{p}<.05]$. Also, more errors were made on the first 100 trials $(\overline{\mathrm{X}}=23.70)$ than on the final 100 trials $(\bar{X}=21.21)[F(1,20)=4.97$, $\mathrm{p}<.05]$. No other differences were obtained.

The results of a Kruskal-Wallis analysis of variance on the ranked percentages of cortical damage (see Figure 1) indicated that there were no significant differences in lesion size between the groups $(H=1.73$, $\mathrm{p}>$.05). In some cases, the temporal lesions involved portions of striate cortex and the parietal lesions involved portions of the somatosensory cortex. These encroachments were minor and no gross locomotor abnormalities were seen in the animals.

Histological analysis of the temporal and parietal lesions showed that two animals with parietal ablations and one animal with a temporal ablation suffered concomitant damage to the dorsal corpus callosum. One animal with a parietal ablation also had damage to the hippocampus. An analysis of retrograde neuronal changes in the thalamus indicated that the parietal ablations were consistently associated with evidence of necrosis and cell loss in the lateral thalamic nucleus. The severity of these retrograde changes appeared to vary directly with lesion size in that large parietal lesions were associated with drastic cell loss in the lateral thalamic nucleus. It is interesting to note that in all cases there were many intact neurons in this nucleus. Temporal ablations were associated with retrograde changes in the lateroposterior thalamic nucleus. These changes were less distinct than those seen in the lateral thalamic nucleus following parietal lesions. The most notable changes were slight to moderate gliosis and cell loss. In two cases, diffuse gliosis was also seen in the junction area of the dorsomedial portions of the lateral geniculate nucleus and the lateroposterior nucleus.

\section{DISCUSSION}

These findings, taken with those from previous studies (Boyd \& Thomas, 1977; McDaniel \& Thomas, 1978; Thomas \& Weir, 1975; Weir \& Thomas, 1975), indicate that a clear dissociation exists between the effects of lesions within the parietal and temporal association cortices in the rat. Lesions of the parietal association cortex appear mainly to disrupt learning dependent upon the use of spatial cues. Lesions of the temporal association cortex produce deficits similar to those seen in primitive primates following extrastriate or temporal association cortex destruction by producing impairments of learning that requires the utilization of visual cues. Boyd and Thomas' (1977) finding that parietal association cortex damage 
disrupts the acquisition of a complex visual discrimination following the acquisition of a simpler visual discrimination utilizing similar cues must be interpreted with caution, as these authors suggested. It is now clear that the parietal deficit in their study did not represent an inability to perceive visual pattern differences but, rather, may have resulted from their training procedures. Also, the hypothesis that rats with parietal association cortex lesions are easily distracted by irrelevant stimuli has not been confirmed. The orientation of the stripe stimuli upon which the relevant cues, orientation of triangles, were superimposed could be viewed as having provided such irrelevant stimuli. However, there was no difference between the nonoperated rats and those with parietal ablations in the number of errors committed while learning this discrimination.

The results of the analysis of errors made by the lesion groups on the discriminations with same vs. different background cues showed that there were more errors made on the discriminations with different backgrounds than on discriminations with identical backgrounds. This result could be interpreted as indicating that the former background of irrelevant stimuli was more distractive across all groups than the latter background. However, no greater deficit was seen in the parietal group than in the other groups. This finding also may imply that rats with parietal cortex lesions are no more distracted by irrelevant stimuli than nonoperated rats. It remains possible that the rats with parietal ablations in Boyd and Thomas' (1977) study were easily distracted or possibly disturbed by the addition of irrelevant pattern stimuli to a previously learned discrimination.

The findings reported here, in conjunction with those reported by Boyd and Thomas (1977), suggest that in order for one to accurately explain the nature of a lesion deficit seen on successive discriminations, it may be necessary to employ control animals that are trained on each individual discrimination. From this it follows, for studies where more than two discriminations are being trained, that optimal interpretations of the research findings would accrue from inclusions of control groups for each sequential combination of discriminations. These statements may be relevant to the interpretations of the functions of the striate and temporal cortices in the visual behavior of the tree shrew made by Diamond and his colleagues (e.g., Diamond \& Hall, 1969; Killackey et al., 1971), which were based, in part, upon the use of a sequential discrimination similar to that used by Boyd and Thomas.

\section{REFERENCES}

Boyd, M. G., \& Thomas, R. K. The effects of posterior association cortex lesions in rats: Mazes, pattern discrimination, and reversal learning. Physiological Psychology, 1977, 5, 455461.

Diamond, I. T., \& Hall, W. C. Evolution of neocortex. Science, $1969,164,251-262$.

Gehring, R. E. Basic behavioral statistics. Boston: HoughtonMifflin, 1978.

Gellerman, L. W. Chance orders of alternating stimuli in visual discrimination experiments. Journal of Genetic Psychology, 1933, 42, 206-208.

Hughes, H. C. Anatomical and neurobehavioral investigations concerning the thalamo-cortical organization of the rat's visual system. Journal of Comparative Neurology, 1977, 175, 311-336.

Killackey, H., \& Diamond, I. T. Visual attention in the tree shrew: An ablation study of the striate and extrastriate visual cortex. Science, 1971, 171, 696-699.

Killackey, H., Diamond, I. T., Hall, W. C., \& Hudgins, G. An ablation study of the tecto-pulvinar-extrastriate system in the squirrel and tree shrew. Federation Proceedings, 1968, 27, 517.

Killackey, H., SNyder, M., \& Diamond, I. T. Functions of striate and temporal cortex in the tree shrew. Journal of Comparative and Physiological Psychology, 1971, 74, 1-29.

Killackey, H., Wilson, M., \& Diamond, I. T. Further studies of the striate and extrastriate visual cortex in the tree shrew. Journal of Comparative and Physiological Psychology, 1972, 81, 45-63.

LASHLEY, K. S. Thalamo-cortical connections of the rat's brain. Journal of Comparative Neurology, 1941, 75, 67-121.

McDaniel, W. F., McDaniel, S. E., \& Thomas, R. K. Thalamo-cortical projections to the temporal and parietal association cortices in the rat. Neuroscience Letters, 1978, 7, 121-125.

McDaniel, W. F., \& Thomas, R. K. Temporal and parietal association cortex lesions and spatial and black-white reversal learning in the rat. Physiological Psychology, 1978, 6, 300-305.

Montero, V. M., Bravo, H., \& Fernández, V. Striateperistriate cortico-cortical connections in the albino and grey rat. Brain Research, 1973, 53, 202-207.

Mountcastle, V. B. The world around us: Neural command functions for selective attention. Neuroscience Research Program Bulletin, 1976, 14 Supplement/April, 1-47.

Robertson, R. T. Thalamic projections to visually responsive regions of parietal cortex. Brain Research Bulletin, 1976, 1, 459-469.

Robertson, R. T., Mayers, K. S., Teyler, T. J., Bettinger, L. A., Birch, H., Davis, J. L., Phillips, D. S., \& Thompson, R. F. Unit activity in posterior association cortex of the cat. Journal of Neurophysiology, 1975, 38, 780-794.

SNyder, M., \& Diamond, I. T. The organization and function of the visual cortex in the tree shrew. Brain, Behavior and Evolution, 1968, 1, 244-288.

Snyder, M., Hall, W. C., \& Diamond, I. T. Vision in the tree shrew (Tupaia glis) after removal of striate cortex. Psychonomic Science, 1966, 6, 243-244.

Thomas, R. K., \& Peacock, L. J. A method of measuring brain lesions. Psychonomic Science, 1965, 3, 184.

Thomas, R. K., \& Weir, V. K. The effects of lesions in the frontal or posterior association cortex of rats on Maze III. Physiological Psychology, 1975, 3, 210-214.

Weir, V. K., \& Thомas, R. K. Failure to find spatial reversal deficits following medial frontal lesions. Bulletin of the Psychonomic Society, 1975, 5, 465-468.
(Received for publication September 19, 1978; revision accepted June 15, 1979.) 